\title{
THE DENSITY OF HUMAN RHODOPSIN IN THE RODS
}

\author{
FRED ZWAS and MATHEW ALPERN \\ Department of Ophthalmology, University of Michigan, Ann Arbor, MI 48104, U.S.A.
}

(Received 11 February 1975; in revised form 20 March 1975)

\begin{abstract}
The density of human thodopsin in the rods has been estimated psychophysically by following the change in the relative quantum sensitivity for rod vision at two wavelengths (one near the peak, the other far off on the long wave tail of the action spectrum) in the dark after a full rhodopsin bleach in a region of a typical $\pi_{0}$ monochromat's retina where cones make no contribution to threshold. Metameric matching and dark adaptation curves yield results (about $0.75 \mathrm{ln}$ units at $500 \mathrm{~nm}$ ) consistent with recent densitometric estimates of Alpern and Pugh (1974) made on the normal retina.
\end{abstract}

\section{INTRODUCTION}

When it first became possible to study the difference spectra of visual pigments in the outer segments of vertebrate photoreceptors under a microscope (Denton and Wyllie, 1955), it was evident that the in vivo concentration of these substances was surprisingly high. There is now considerable psychophysical evidence to substantiate the validity of such observations for human cones (Brindley, 1955; Enoch and Stiles, 1961; Walraven, 1962; King-Smith, 1973; Miller, 1973). However, following Hecht, Shlaer and Pirenne (1942) it is usually assumed that the density of rhodopsin in human rods is small (about $0.23 \mathrm{ln}$ units at the wavelength of peak absorbance). This assumption has now been undermined by the recent densitometry analysis of Alpern and Pugh (1974) which suggests that human rhodopsin concentration in vivo is about three times higher than this (i.e. more or less what is found in cones). Can psychophysical evidence be obtained which validates this estimation?

When a visual pigment is in high concentration, in vivo, its action spectrum will depend upon the fraction of the total complement in the unbleached state. Immediately after a full bleach, the fraction of unbleached molecules is very low and a light of wavelength near the tail of the absorption spectrum should require relatively more quanta to produce some constant physiological effect in comparison with a light of wavelength near the peak of the absorption spectrum than will be the case when all the pigment has regenerated. In effect then as rhodopsin regenerates in the dark, rod sensitivity tested at the $\lambda_{\max }$ becomes progressively reduced relative to that at the "tail". This effect is known as "self screening".

Even before the absorption spectrum of human rhodopsin was on firm experimental grounds, Nagel (1911) seems at least partly to have appreciated this fact. In a posthumous unrefereed report in the Appendix to the 3rd Edn. of Helmholtz' Physiolog ical Optics (the editorial preparation of which he had begun but had not completed at his untimely death), he describes psychophysical evidence of this kind obtained by Stegmann and himself on the rod vision of both normals and one typical $\pi_{0}$ monochromat. On the other hand, de Vries (1946) failed completely to confirm these observations on normal subjects.
Evaluating this contradiction is complicated by the fact that in the dark after a full bleach almost all rhodopsin in the normal retina has regenerated before rod sensitivity exceeds that of cones and rod vision becomes testable (Rushton, 1961). We have, therefore, analyzed the problem anew in a region of the retina of one typical $\pi_{0}$ monochromat with no measurable cone function. The procedure was to compare rod spectral sensitivity at two wavelengths, one $(500 \mathrm{~nm})$ near the peak; the other, $(632.8 \mathrm{~nm})$ far off on the low frequency "tail", of the absorption spectrum under different bleaching circumstances (and, therefore, different rhodopsin concentrations).

\section{The subject}

The $S$ was a 25 -yr-old patient referred to this laboratory by Dr. H. F. Falls with the diagnosis of typical $\pi_{0}$ monochromacy. She had been under his care for the last $7 \mathrm{yr}$ and had been studied by him and in this laboratory intermittently during that interval with no change in signs and symptoms. These included: a purely scotopic spectral sensitivity curve irrespective of how it is tested, a central scotoma, $4^{\circ}$ dia, a minute pendular nystagmus, visual acuity R.E. $20 / 200+1$, L.E. 20/100-1 which could not be improved with the spectacle correction for her small amount of 'simple hyperopic astigmatism. She reads $J$. $O$. at nearby holding the card very close to her eyes. The slit lamp examination was free of evidence of any pathological abnormalities and the ophthalmoscopic examination failed to reveal any evidence of macular disease; the discs were normal as were the vascular trees. She is totally color blind. Retina densitometry of the retina area used for central fixation revealed only thodopsin (Alpern, 1974). Rod saturation at moderately bright backgrounds (equal to or greater than 1000 scotopic td) results in a "photophobia" but exhibits no evidence of the long term (up to $2 \mathrm{hr}$ ) "purblindness" described by Walls and Heath (1954) in a somewhat similar subject after such exposures. Nor do we confirm for her the observations of Sakitt (1973) on a so-called rod monochromat that thresholds after bleaching saturate so that however intense the test it remained invisible until about $75 \%$ of the thodopsin had regenerated. Rather, in general, the constraints on visibility early in the dark after a full bleach for this subject appeared to be those imposed by the upper limit of the intensity of the test flash.

Occasional ("rhodopsin") cones can be found in some regions of the retinas of monochromat eyes. Psychophysically they are revealed by a double-branched dark adaptation curve measured after a full bleach and by an increment threshold curve which does not show the characteristic rod saturation. We have deliberately tested a region of this subject's retina which manifests neither of 


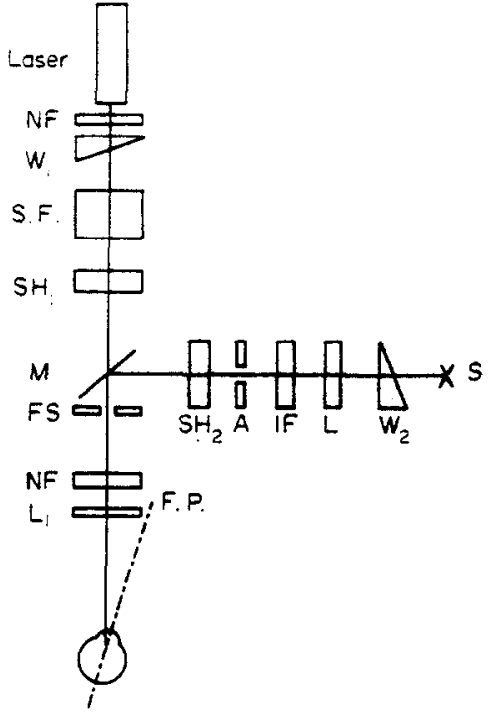

Optical system

Fig. 1. Schematic drawing of the optical system as viewed from above (not to scale). Light from two sources, a Spectra Physics helium-neon laser $(\lambda=632.8 \mathrm{~nm})$ and $a$ tungsten filament $S$ are mixed together at $M$. The field stop, FS, is located at primary focal plane of $L_{1}$ (a type I Kodak Ektar $f 2.5,155 \mathrm{~mm}$ focal length lens); the aperture stop, A, in Channel 2 and the pinhole of the spatial filter, S.F., (Channel 1) are equidistant from $M$ and both are imaged in the subject's pupil by $L_{1}$. The Baird-Atomic interference filter, IF, renders Channel 2 monochromatic at $\lambda=500 \mathrm{~nm}$ with a half band width of $10 \mathrm{~nm} . F$. P. is the fixation point: $\mathrm{SH}_{1}$ and $\mathrm{SH}_{2}$ are Uniblitz (Vincent Associates, Rochester, N.Y.) shutters: $W_{1}, W_{2}$ are "neutral" wedges and NF "neutral" density filters. The lens, $L_{2}$, images the tungsten filament on $A$.

these evidences of cones. In particular, log threshold in the dark after a bright bleach follows the time course of a simple exponential curve and the increment threshold curve saturates at backgrounds of about $10^{3}$ scotopic td (with a semi-saturation constant of $300 \mathrm{~h} \nu$ absorbed rod $^{-3}$ flash $^{-1}$ ).

\section{METHOD}

Figure 1 is a line drawing of the apparatus. Monochromatic lights from two separate sources (one, a laser providing $632.8 \mathrm{~nm}$, the other, a tungsten filament attenuated by an interference filter transmitting $500 \pm$ $10 \mathrm{~nm}$ ) were brought together at the mirror $M$. The intensity of each light beam was reduced by balanced Inconel Wedges $\left(W_{1}\right.$ and $\left.W_{2}\right)$ and by "neutral" Inconel filters, all calibrated with a Beckman Acta II spectrophotometer. The test target was viewed by a patch of retina $8^{\circ}$ temporal from the fixation point. The circular stop FS requlated the field size; in testing, it was $3^{\circ}$ and in bleaching, $8^{\circ}$ dia. Bleaching was provided by the tungsten source after the interference filter (IF) was removed from the beam; it then produced $6.5 \mathrm{log}$ td and was exposed for 2 min. This was sufficient to bleach all measurable rhodopsin in the patch of retina illuminated by the focused light.

The subject's head was fixed by biting into a wax dental bite. The image of the laser aperture stop in the plane occupied by her pupil was $0.5 \mathrm{~mm}$; that of the tungsten source was $1.0 \mathrm{~mm}$. These two images were carefully centered on each other. They were positioned in the center of the pupil while she fixated. Widely dilating her pupil with one drop of $1 \%$ cyclogy $\left.\right|^{\oplus}$ obviated eclipsing either beam by the iris due to the small nystagmus movements characteristic of the fixation of such subjects The test flashes were exposed for $50 \mathrm{msee}$ once a second by two shutters. one in each beam, controlled by Grass Physiological stimulators. This permitted the experimenter to determine the wavelength under test at any moment in the dark after bleaching. In brightness matching, both channels were simultaneously exposed: in this case, only a single shutter mounted in the common beam was used.

All components of the optical system were mounted on saddles fixed to triangular optical bench rails clamped to a 1.5-in. 8-ply plywood table top. Three different mirrors. each mounted in a separate saddle, served the function in turn of the mirror at M. In bleaching, this was a full silvered mirror reflecting all the light from the tungsten source and transmitting none of the laser light. After the bleach, the saddle containing this mirror was removed and replaced with one or the other of the remaining two. In those experiments in which threshold was measured. its replacement contained a beam mixer which reflected about $30 \%$ of the incident tungsten light and transmitted about $30 \%$ of the incident laser light. In brightness matching, the third saddle was used. It contained a full surface mirror, the edge of which vertically bisected the field of view into two equal parts: (i) that light reflected at the mirtor from the tungsten source and (ii) that part of the laser beam unobstructed by the mirror. Each of these mirrors had been precisely aligned prior to the experiment. Since the location of the proper position of the replacing saddle was easily identified and achieved in the dark, these mirrors were readily interchanged manually during the run without disturbing the optical alignment.

The maximum exposure of the laser beam in these experiments was $2.95(10)^{-3} \mathrm{~W} / \mathrm{cm}^{2}$ at the retina. This is more than $2.5 \mathrm{log}$ units dimmer than the minimum intensity of a $50 \mathrm{msec}$ flash for damage to any ocular tissue (Peppers and Hammond, 1969).

\section{PART I. METAMERIC MATCHING}

\section{Procedure}

The test was divided into two half fields: on the left. the monochromatic green light, and the laser monochromatic red light on the right. Both beams were exposed together (for $50 \mathrm{msec}$ ) and the observer's task was to adjust the red wedge so that the two fields matched under two different observation conditions (and, therefore, two different presumed rhodopsin concestrations): (1) after $30 \mathrm{~min}$ in the dark $[c=\beta(\lambda) / \alpha(\lambda) l]$ and (2) in the dark immediately after a full rhodopsin bleach $(c=0)$. In fact, no measurements were possible immediately after the bleach; it required a full minute at the least before the bipartite field was visible. The comparison was, therefore, between the matches made between 60 and 150 sec after the bleaching and those obtained after complete dark adaptation.

The red and green intensities were first matched in full dark adaptation by placing neutral filters in the common path immediately behind the lens $L_{1}$ and then by setting the wedges, $W_{1}$ and $W_{2}$, somewhere in the middle of their respective ranges. The final match was always established with the red wedge $W_{1}$ and only it was adjusted during the experiment before or after bleaching. Following bleaching, both beams were made considerably brighter by removing neutral filters from the common path. Throughout brightness matches were established between lights always approx $1 \log$ unit above threshold.

\section{Analysis}

It is assumed that: (i) only absorbed quanta enter into the photochemical events which initiate vision (ii) Beers and Lambert's Law adequately describes quantum absorption in the rods and (iii) equal quanta absorptions cause equal visual effects 
independent of wavelength: This last assumption (Naka and Rushtons' (1966) univariance) applies to one photoreceptor cell or to groups of such cells, all of which have the same visual pigment. In the typical $\pi_{0}$ monochromat here under study, we have deliberately sought out that region of her retina where dark adaptation and increment threshold measurements show that the latter is clearly the situation.

When two half fields of wavelengths $\lambda_{1}$ and $\lambda_{2}$ are illuminated with lights of intensity $I_{1}$ and $I_{2}$ respectively, so adjusted that they appear identical:

$$
I_{1} T_{1}\left[1-\exp \left(-\alpha_{1} l c\right)\right]=I_{2} T_{2}\left[1-\exp \left(-\alpha_{2} l c\right)\right] .
$$

In this equation $T_{1}$ and $T_{2}$ represent the respective transmissivities of the ocular media at $\lambda_{1}$ and $\lambda_{2}$.

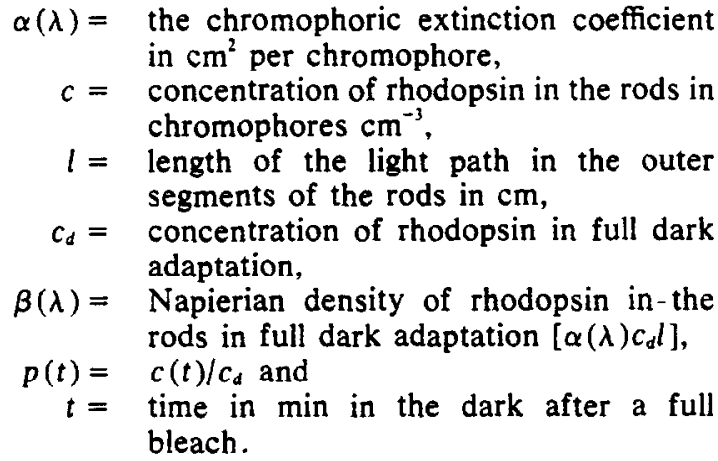

The wavelengths of the two test lights here under consideration were so selected that according to the C.I.E. scotopic spectral sensitivity curve

$$
\left[1-\exp \left(-\beta_{1}\right)\right] /\left[1-\exp \left(-\beta_{2}\right)\right]=2 \cdot 7(10)^{-3} .
$$

Under these conditions, it is reasonable to assume that in the series expansion

$$
1-\exp \left(-\beta_{1}\right)=\beta_{1}-\beta_{1}^{2} / 2 !+\beta_{1}^{3} / 3 !-\beta_{1}^{4} / 4 ! \ldots
$$

$\beta_{1}$ is so small that the higher order terms can safely be neglected. Accordingly, in full dark adaptation equation (1) reduces to

$$
I_{1} T_{1} / I_{2} T_{2}=\left[1-\exp \left(-\beta_{2}\right)\right] / \beta_{1} .
$$

After a full bleach $c \rightarrow 0$, so that the matching intensities at the two wavelengths $I_{1}^{\prime}, I_{2}^{\prime}$ are related by

$$
I_{1} T_{1} / I_{2}^{\prime} T_{2}=\beta_{2} / \beta_{1}
$$

so that

$$
I_{1} I_{2}^{\prime} / I_{2} I_{1}^{\prime}=\left[1-\exp \left(-\beta_{2}\right)\right] / \beta_{2} .
$$

If $k$ is the density of the "neutral" filters in the common beam in full dark adaptation which were removed for the matches after the bleach and $W_{1}$ is the transmissivity of the red wedge (plus any transmissivity differences at the two wavelengths in the "neutral" filters removed from the common beam), then

$$
\log I: / I:=k
$$

and

$$
\log \left(I_{1} / I_{1}^{\prime}\right)=k+\Delta \log W_{1} .
$$

Hence from equation (2)

$$
\Delta \log W_{1}=\log \left[1-\exp \left(-\beta_{2}\right)\right] / \beta_{:} .
$$

\section{Results}

The results of this experiment (repeated five times) are summarized in Table 1 . The first column gives the average ( \pm 1 S.D.) of red wedge density in the interval between $1 \mathrm{~min}$ and $2.5 \mathrm{~min}$ in the dark after a full bleach. The second column gives the average density ( \pm 1 S.D.) of the same wedge settings after 30 min (corrected for non neutrality of the common filters added). It is seen that in each of the five sets of experiments relatively more red light was required for the match immediately after bleaching than was the case in full dark adaptation. This is the expected result if the rhodopsin in the monochromat's rods was in considerable density. The densities $\beta$ : (i.e. at $500 \mathrm{~nm}$ ) calculated from these measurements according to equation (3) are given in the third column.

Student's t-test was utilized to evaluate the significance of the differences in the settings of the red wedge (the degrees of freedom for each experiment being listed in the fourth column in the table).

These results show considerable variability in the estimation of $\beta_{2}$ from one run to the next. Furthermore, only two of the five estimations in Table 1 differ significantly from zero.

The reasons for these difficulties are not hard to find. Rushton (1961) and Alpern (1971 a,b) have independently shown that after a full bleach rhodopsin regenerates in vivo according to the equation

$$
p(t)=c(t) / c_{d}=[1-\exp (-t / \tau)],
$$

in which $\tau$ is the time constant of regeneration of rhodopsin (about $9.33 \mathrm{~min}$ for the patch of retina under consideration here).

Since, in the above experiments, the first measurements were made between 1 and $2.5 \mathrm{~min}$ in the dark, the rhodopsin concentrations at these instances were not zero but between 0.1 and 0.235

Table 1. Brightness matches (red wedge densities) at beginning and end of dark adaptation. Typical $\pi_{0}$ monochromat

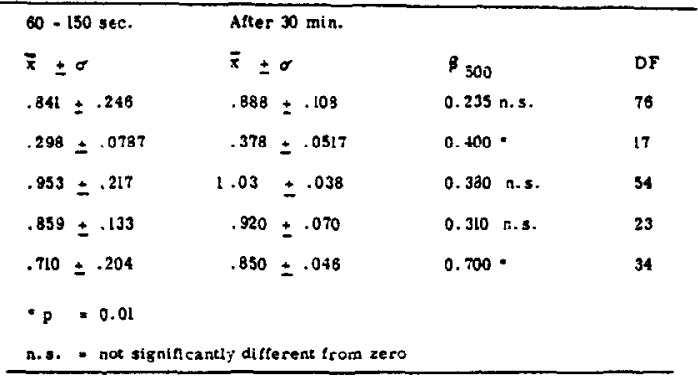


times its full dark concentration. Part of the variability in these results must be due to the fortuitous set of circumstances under which the initial measurements were completed sooner after the bleach in one run than in another. Since no settings could be made near zero concentration, the results in Table 1 can be regarded only as estimates of the lower limit of rhodopsin density.

To obviate these problems, an attempt was made to extrapolate the red wedge setting to the value it would have had at the moment $(t=0)$ the bleaching light was extinguished (when the rhodopsin concentration was known to be very nearly zero). The calculation was strengthened by making brightness matches throughout the $30 \mathrm{~min}$ or so in the dark after bleaching while rod sensitivity is changing (rather than only at the very beginning and at the very end of adaptation as was done above). It is seen from equation (3) that

$$
\begin{aligned}
\Delta \log W_{1}(t)= & \log \left\{\left[1-\exp \left(-\beta_{2}\right)\right.\right. \\
& \left.\left.\times\left(1-\mathrm{e}^{-t / \tau}\right)\right] /\left[\beta_{2}\left(1-\mathrm{e}^{-t / \tau}\right)\right]+a\right\} .
\end{aligned}
$$

In this equation, $a$ is the asymptotic value of $\log W_{1}$ when $t=\infty$.

\section{Experiment}

As soon as the test target was visible in the dark after a full bleach, the observer made brightness matches by adjusting the red wedge. The process was repeated over and over until the values of her settings leveled off after $30 \mathrm{~min}$ or so. The experimenter recorded the time and the red wedge setting of each adjustment and periodically added a neutral filter to the common beam so that the intensity of the green field always hovered around a level about $1 \log$ unit above its absolute threshold. The experiment was repeated four times on a single day.

The results of each run were analyzed by computer to determine the values of $\beta$, and $a$ in equation (5a) which minimized the squares of the deviations from the curve described by this equation (given $\tau=9.33 \mathrm{~min}$ ). The best estimates of $\beta_{2}$ ( \pm 1 S.D.) resulting from the individual runs are summarized in the insert in Fig. 2.

The value for $a$ is a function of more or less arbitrary decisions of the experimenter regarding the neutral filters in the common beam and differed from run to run. In pooling the results from the four runs, the individual sets of results were vertically shifted by computer to provide the best overall fit to equation (5a). The individual data points in Fig. 2 are the results from each run plotted as $\Delta \log W$ after this shifting. The smooth curve drawn through these results is the least squares estimate of the best fit to equation ( 5 ). It yields an average estimate of $\beta_{2}=0.733 \pm 0.093$ (mean \pm 1 S.D.). Although there is considerable scatter in Fig. 2, the ordinate scale of that figure is very enlarged. The density estimate obtained is significantly different from zero and in substantial agreement with the Alpern and Pugh densitometric value, hence considerably higher than that predicted from Hecht, Shlaer and Pirenne (1942). The right hand ordinate scale in Fig. 2 shows how the value of $\beta_{2} p(t)$ increases with time in the dark as the rhodopsin concentration

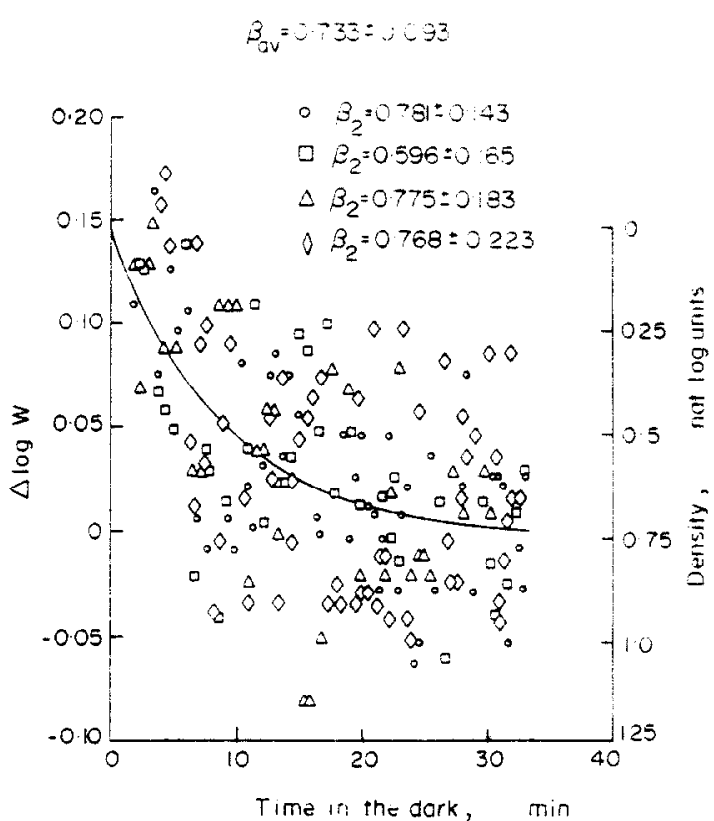

Fig. 2. Changes in the red wedge setting required for brightness matches as a function of time in the dark after a full bleach. The four different symbols represent results from four experimental repetitions completed in a single day. The experimental results from each run were utilized to generate by computer those values $\beta_{2}$ and $a$ which, when substituted in equation ( $5 \mathrm{a})$, minimized the squares of the deviations from the theoretical curve (given $\tau=9.333 \mathrm{~min}$ ). The points plotted in this figure have been vertically shifted so that all results have a common asymptote. The average estimate of $\beta_{2}=0.733 \pm 0.093$. The table insert gives the estimate of $\beta_{2}$ from each run. The ordinate scale on the right shows how density at $500 \mathrm{~nm}$ increased as rhodopsin concentration returned to the full dark value after the bleach.

changes. Comparing the two ordinate scales in this figure emphasizes further the difficulty in obtaining accurate psychophysical estimates of the rhodopsin density from individual expreiments. Given the high density of 0.733 , the total range of $\Delta \log W$ is only $0.15 \mathrm{log}$ units. The precision of individual brightness matches in rod vision is typically only $0.05-0.10 \log$ units.

\section{PART II. ABSOLUTE THRESHOLD CHANGES} IN THE DARK

For this reason, a second psychophysical estimate of the density of rhodopsin in this monochromat's rods was obtained by measuring threshold changes during dark adaptation.

\section{Procedure}

After the bleach, the subject sat in the dark and observed the flashing green light set at its maximum intensity signalling the moment it became visible. The experimenter then switched the shutter so that the red test was flashing and the subject adjusted the red wedge for threshold. The experimenter then switehed the shutters so that the green test was flashing and recorded both the red wedge setting and the moments of its adjustment from the stop watch reading; meanwhile, the subject adjusted the green wedge for threshold. The experiment proceeded in this way alternatively testing for green then red throughout some $33 \mathrm{~min}$ in the dark after the full bleach. The shaft on which the green wedge rotated was geared to a 
potentiometer which was wired to the $Y$-axis of a Hewlett-Packard 7035B $X-Y$ plotter, the $X$-axis of which was driven by a $17108 \mathrm{~A}$ External Time Base reset at zero at the moment the bleaching light was extinguished. In this way, the subject automatically noted the green wedge settings by activating a signal marker; the experimenter recorded both the moment and the value of the red wedge settings while continuously switching the shutters from one beam to the other throughout the run. As before. neutral filters were added to the common beam whenever they were needed.

\section{Analysis}

The assumptions made in the derivation of equation (2) are as appropriate for the analysis of dark adaptation measurements as they are for brightness matching. In using this equation in this context, $I_{\lambda}$ represents the threshold intensity at wavelength $\lambda_{1}, \lambda_{2}$ after infinite time in the dark and $I_{\lambda}^{\prime}$ the threshold intensities immediately after a full rhodopsin bleach.

In practice, $I_{\lambda}, I_{\lambda}^{\prime}$ must be estimated by extrapolations. To this end, we take advantage of the fact (Rushton, 1961; Alpern, 1971 a,b) that a single exponential curve describes the time course of the recovery when log threshold is plotted as a function of time in the dark after a full bleach and the test is made of a region of the monochromat's retina in which rods alone contribute to threshold. A computer program (Chandler, 1965) was utilized to fit equations of the form $y=A+B \exp (-t / \tau)$ [in which $y$ is $\log$ threshold (in quanta flash ${ }^{-1}$ )] to the measured dark adaptation results so as to minimize the square of the deviations from the curves. The values of $A=\log I_{\lambda}$ and $A+B=\log I_{\lambda}^{\prime}$ were then determined directly from these best fitting exponential curves.

\section{Results}

The results of four experimental repetitions are shown in the usual way in Fig. 3. In the major part of this figure, each plotted point is a single measurement and the smooth curves drawn through them are the best fitting exponential recovery curves obtained from the computer analysis. The values of $I_{1}, I_{1}^{\prime}, I_{2}$ and $I_{2}^{\prime}$ obtained from these curves when substituted into equation (2) yield the estimate of $\beta(500)=0.787$ in good agreement with that obtained from brightness matching. In order to appreciate how subtle the difference in recovery is which yields this estimate of high density, the two computer generated curves alone (without the data points) are replotted to a smaller scale in the inset with the asymptotic value of the curve for the 632.8-nm test (dashed curve in the figure) arbitrarily equated to that of the $500-\mathrm{nm}$ test (solid curve). The two curves are virtually indistinguishable except for the first $9 \mathrm{~min}$ or so in the dark.

A word regarding the curve fitting in Fig. 3 used to obtain $I_{\lambda}^{\prime}$ by extrapolation is in order. This procedure is based upon the observation that simple exponential curves provide the best (albeit purely empirical) description of the experimental results. On theoretical grounds, if $p=0$ at $t=0$ there is the difficulty that there would be no rhodopsin molecules available to catch the quanta in the test flash whose intensity must be infinitely large, though the exponential extrapolations in Fig. 3 to $t=0$ yield log $\left[I^{\prime} / I_{-}\right]=A+B$. Hollins and Alpern (1973) developed a

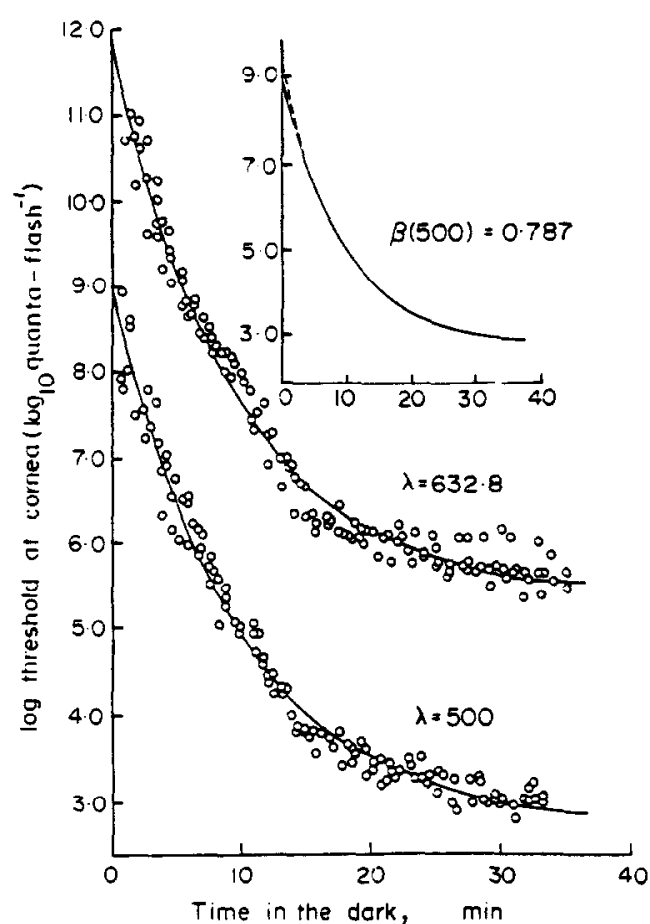

Fig. 3. Simultaneous rod dark adaptation threshold measurements for a red and a green test as a function of time in the dark after a full bleach. The results of four experimental repetitions are presented, and the smooth curves drawn are the best fitting exponential functions obtained by a computer analysis. The equations for the two curves are

$$
y_{2}=2.803+6.323 \exp (-t / 9 \cdot 54)
$$

and

$$
y_{1}=5 \cdot 486+6 \cdot 486 \exp (-t / 9 \cdot 34) .
$$

The parameters from these equations (i.e. $\log I_{\mathrm{i}}=11.972$, $\log I_{2}^{\prime}=9.126, \log I_{1}=5.486$ and $\log I_{2}=2.803$ ) when substituted into equation (2) give an estimate for the density (at $500 \mathrm{~nm}$ ) equal to 0.787 . To appreciate the small difference which yields this value, the two computer generated curves are replotted in the inset with the curve for the red test (dotted curve in figure) arbitrarily shifted until its asymptote was identical to that of the curve for the green test.

more general equation to deal with this difficulty for foveal $\pi$, cones. Their equation,

$$
\log \left[I(t) / I_{x}\right]=k_{1}\left(1-\mathrm{e}^{-\beta}\right)(1-p) /\left(1-\mathrm{e}^{-\boldsymbol{- \alpha p}}\right),
$$

(in which $k_{1}$ is a constant) did not, however, describe their results more successfully than simple exponential curves, such as the ones drawn in Fig. 3. We have applied equation (6) to the results in Fig. 3, but the best fitting curves of this kind are about $15 \%$ poorer fits than those shown in the figure, the root mean square deviation of the measurements from which are relatively small $(0.22$ and 0.23 respectively). For this reason, and because all known theories which have attempted to relate log rod threshold elevation with fraction of unregenerated pigment after a full rhodopsin bleach are clear oversimplifications (Alpern, 1971a; Duffy, 1971; Pugh, 1972), we have selected the superior empirical description. Conceptually this assumes that $p$ only approaches zero at $t=0$. It should be appreciated, however, that the application of equation (6) does allow an estimation of $I_{i}^{\prime} / I_{2}^{\prime}$ even though $I_{i}^{\prime}$ approaches infinity at $t=0$ and that the resulting value of 
$\beta_{2}$ obtained from the results in Fig. 3 with this estimation $(1 \cdot 28)$ is even higher than that obtained from the curves drawn in Fig. 3. Hence, the decision to use the purely empirical equation may prove ultimately to have yielded only a lower limit estimate (unconventionally high as it is) of the density of rhodopsin in this monochromat's rods.

\section{Discussion}

The results of the two different methods used here to study rhodopsin density psychophysically in a typical $\pi_{0}$ monochromat's retina are in good agreement with the estimates of rhodopsin density made with retinal densitometry on the normal retina by Alpern and Pugh (1974). These converging lines of evidence for a value much higher than that given by Hecht et al. are summarized in Table 2 .

The results in this table are also in good agreement with microspectrophotometric measurements of rhodopsin density in individual rods, the most recent of which (MacNichol, Feinberg and Harosi, 1973) are considerably larger $(0.0175 \pm$ 0.0015 common log units $/ \mu \mathrm{m})$ than Liebman's (1962) earlier results $(0.013 \pm 0.0002)$. For a rod outer segment $25 \mu \mathrm{m}$ long, the respective optical densities corresponding $(0.4375-0.325)$ are a good deal higher than the macrospectrophotometric measurements of Bauman and Bender (1973) of 0.1, in part no doubt because the latter estimates are diluted by stray light (Alpern and Pugh, 1974).

In view of the results illustrated in Fig. 3, it is not surprising that de Vries (1946) found no evidence for the effect of bleaching on rod spectral sensitivity of his own (normal) eye. It is now known that in light adaptation rod vision saturates before any significant rhodopsin is bleached (Aguilar and Stiles, 1945) and the dark adaptation curves in Fig. 3 clearly show that after a full bleach the rod dark adaptation curves for a red and green test would have superimposed before rod sensitivity exceeded that of cones in the normal peripheral retina. It is for this reason that these measurements had to be made on a typical $\pi_{0}$ monochromat retina to demonstrate the subtle effect of high density on rod spectral sensitivity.

De Vries' selection of $580 \mathrm{~nm}$ for a long wave test $\left(\lambda_{1}\right)$ was also not judicious for obtaining evidence for high in vivo rhodopsin density. His value of $\left[1-\exp \left(-\beta_{1}\right)\right] /[1-$ $\left.\exp \left(-\beta_{2}\right)\right]$ is only 0.121 . With the two wavelengths used here, this ratio is 0.0027 but still only very small distinctions in the earliest moments in the dark after a full bleach were found.

Although our conclusion is qualitatively, at least, identical to that of Stegmann and Nagel, we do not know how to deal adequately with the experimental results which led them to it:

"If, for example, after being dark-adapted for a period of 5 to $10 \mathrm{~min}$ 'twilight matches' were made

Table 2. Mean estimate of rhodopsin density $(\lambda=$ $500 \mathrm{~nm}$ )

\begin{tabular}{|c|c|c|}
\hline Mathod: & Deantey & Seasity \\
\hline xatura & $\begin{array}{l}\text { Logartehmse } \\
\text { whitts }\end{array}$ & $\begin{array}{c}\text { Comon lojartetrate } \\
\text { entes }\end{array}$ \\
\hline Densicometry (Alpern s Pugh, 197:) & 0.788 & 0.342 \\
\hline Brightaess zatching & 0.733 & 2.313 \\
\hline Dark adaptartion turve: & 0.787 & 3.142 \\
\hline
\end{tabular}

between a spectral orange and blue-green (both of which would, of course, look colourless), and if then this match was considered by the same eye after it had been dark-adapted for a much longer time, it ceased to be valid: the light of longer wavelength (orange) looked the brighter of the two. and it was necessary to reduce its intensity to three-fourths of what it had been."

We have been unable to find any more extensive description of these experiments, but this result appears compatible with those described here only if the kinetics of the rhodopsin in the eyes making these observations were radically different from those of any human eyes studied so far with the retinal densitometer.

Acknowledgements-Assisted by a grant EY-00197 from the National Eye Institute to Mathew Alpern, by a FIGHT FOR SIGHT Postdoctoral Research Fellowship (F 257) to Fred Zwas, FIGHT FOR SIGHT, INC., New York City, and by an unrestricted grant to the Department of Ophthalmology of the University of Michigan from Research to Prevent Blindness, Inc., New York City.

We thank our subject, Karen Koeman, for her patience and Gilbert B. Lee, Drs. H. F. Falls. E. Daniels, F. Maaseidvaag, and S. Miller for their technical assistance and suggestions.

\section{REFERENCES}

Aguilar M. and Stiles W. S. (1954) Saturation of the rod mechanism of the retina at high levels of stimulation. Optica Acta I, 59-65.

Alpern M. (1971a) Rhodopsin kinetics in the human eye. $J$. Physiol., Lond. 217, 447-471.

Alpern M. (197 1b) The effect of a bright light flash on dark adaptation of human rods. Nature, Lond. 230,394-396.

Alpern M. (1974) What confines in a world without color? Investve Ophth. 13, 647-674.

Alpern M. and Pugh E. N. (1974) The density and photosensitivity of human rhodopsin in the living retina. J. Physiol., Lond. 237, 341-370.

Bauman C. H. and Bender S. (1973) Kinetics of rhodopsin bleaching in the isolated human retina. J. Physiol., Lond. 235, 761-773.

Brindley G. S. (1955) A photochemical reaction in the human retina. Proc. phys. Soc. 68-B, 862-870.

Chandler J. P. (1965) STEPIT. Quantum chemistry program exchange. Dept of Chemistry, Indiana Univ.. Bloomington.

de Vries H. P. (1946) Concentration of the visual purple in the human eye. Nature, Lond. 158, 303.

Denton E. J. and Wyllie J. H. (1955) Study of the photosensitive pigments in the pink and green rods of the frog. J. Physiol., Lond. 127, 81-89.

Duffy W. J. (1971) The time course of rod dark adaptation following two kinds of rhodopsin bleaches. Ph.D. thesis, Univ. of Michigan.

Enoch J. M. and Stiles W. S. (1961) The colour change of monochromatic light with retinal angle of incidence. Optica Acta 8, 329-358.

Hecht S., Shlaer S. and Pirenne M. (1942) Energy, quanta and vision. J. gen. Physiol. 25, 819-840.

Hollins M. and Alpern M. (1973) Dark adaptation and visual pigment regeneration in human cones. $J$. gen. Physiol. 62, 430-447.

King-Smith P. E. (1973) The optical density of erythrolabe determined by retinal densitometry using self-screening methods. J. Physiol., Lond. 230, 535-549.

Liebman P. A. (1962) In situ microspectrophotometric studies on the pigments of single retinal rods. Biophys. J. 2, 161-178. 
MacNichol E. F., Jr., Feinberg R. and Harosi F. I. (1973) Colour discrimination processes in the retina. Colour 73: Proc. 2nd Congr. of the Int. Colour Ass, pp. 191-251. Adam Hilger, London.

Miller S. S. (1972) Psychophysical estimates of visual pigment densities in red-green dichromats. J. Physiol., Lond. 223, 89-107.

Nagel W. (1924) In H. V. Helmholtz' Physiological Optics (English translation of the 3rd Edn.), Vol. II. p. 390. Opt. Soc. Am., New York.

Naka K. I. and Rushton W. A. H. (1966) S-potentials from luminosity units in the retina of fish (Cyprinidae). $J$. Physiol. Lond. 185, 587-599.

Peppers N. A. and Hammond A. H. (1969) Laser damage thresholds for ocular tissue. Am. ind. Hyg. Ass. J. 130. 218-225.

Pugh E. N., Jr. (1972) The paradoxical elevation of rod threshold after flash bleaching of the human retina. Ph.D. thesis, Univ. of Michigan.

Rushton W. A. H. (1961) Rhodopsin measurement and dark adaptation in a subject deficient of cone vision. $J$. Physiol. Lond. 156, 193-205.

Sakitt B. (1973) Rod saturation. Proc. ARVO, p. 99.

Walls G. L. and Heath G. G. (1954) Typical total color blindness reinterpreted. Acta ophthal. 32, 254.

Walraven P. L. (1962) On the mechanisms of color vision. Inst. for Perception RVO-TNO, Soesterberg, Netherlands, 1-94. 